\title{
VHF scintillations, orientation of the anisotropy of F-region irregularities and direction of plasma convection in the polar cap
}

\author{
E. D. Tereshchenko ${ }^{1}$, N. Yu. Romanova ${ }^{1}$, and A. V. Koustov ${ }^{2}$ \\ ${ }^{1}$ Polar Geophysical Institute, Russian Academy of Sciences, 15 Khalturina, 183010 Murmansk, Russia \\ ${ }^{2}$ Institute of Space and Atmospheric Studies, University of Saskatchewan, Saskatoon, Canada
}

Received: 13 November 2007 - Revised: 7 March 2008 - Accepted: 14 March 2008 - Published: 18 June 2008

\begin{abstract}
Scintillation data recorded at the polar cap station Barentsburg are shown to occasionally exhibit two or more peaks in the latitudinal profiles of the amplitude dispersion. Comparison with concurrent SuperDARN radar convection maps indicates that multiple peaks occur when Barentsburg is located within the area of strong changes in the plasma flow direction. When parameters of the ionospheric irregularities are inferred from the scintillation data, the orientation of the irregularity anisotropy in a plane perpendicular to the magnetic field is found to coincide well with the $\boldsymbol{E} \times \boldsymbol{B}$ flow direction, individually for each peak of the scintillation data. The differences were found to be mostly less than $20^{\circ}$ for a data set comprised of 104 events. The conclusion is made that analysis of scintillation data allows one to infer the direction of plasma flow with a certain degree of detail.
\end{abstract}

Keywords. Ionosphere (Auroral ionosphere; Ionospheric irregularities)

\section{Introduction}

VHF radio signals arriving from a low-orbiting satellite to a receiver on the ground often experience rapid fluctuations, termed scintillations (Aarons, 1982). Such fluctuations occur because radio waves are scattered/refracted by the F-region ionospheric irregularities with the scale of several hundred meters. While scintillations cause limitations to communication with satellites, they are useful for extracting information on various parameters of the ionosphere (Moorcroft and Arima, 1972; Martin and Aarons, 1977; Fremouw et al., 1977; Rino et al., 1978; Rino and Livingston, 1982; Gailit et al., 1982; Eglitis et al., 1998; Milan et al., 2005). In a series of papers, Tereshchenko et al. (1999, 2000a, b, 2002, 2004,2005 ) showed that one can infer a statistically averaged

Correspondence to: N. Yu. Romanova

(romanova@pgi.ru) shape of F-region anisotropic irregularities by analyzing both the phase and amplitude of a satellite signal received on the ground. It has been demonstrated that such irregularities are strongly stretched along the magnetic field lines and somewhat less in a plane perpendicular to the magnetic field. In addition, the authors hypothesized and then presented evidence that the orientation of the cross-field anisotropy of Fregion irregularities (in a plane perpendicular to the magnetic field) is aligned with the $\boldsymbol{E} \times \boldsymbol{B}$ plasma drift (convection), a property that can be used for estimates of plasma flow direction at high latitudes (Tereshchenko et al., 2000a, b, 2002, 2005).

Tereshchenko et al.'s method in its original form is based on an assumption that F-region irregularities exist throughout a uniform infinite layer at the height of $\sim 300 \mathrm{~km}$. As a satellite travels roughly along a magnetic meridian near the zenith of a receiver, the measured value for the variance of the logarithm of the relative amplitude $\sigma_{\chi}^{2}$ of the signal exhibits a strong peak at a certain latitude, and this peak always can be matched with the theoretically expected curve for the variation of $\sigma_{\chi}^{2}$ with latitude. Minimisation of the difference between the model and the data allows one to obtain the 3-D parameters of the F-region irregularities. Analysis shows that for typical satellite-receiver geometries the theoretical profile of $\sigma_{\chi}^{2}$ has a single peak in latitude. Moreover, the location of the peak is not very sensitive to the choice of the thickness of the irregularity layer and its height; it only shifts by not more than $\sim 1^{\circ}$. In this sense, the derived irregularity parameters are unique ones for a given set of measurements.

It is clear that the assumption of a layer filled with irregularities of the same shape and uniformly distributed within the layer is simplistic for a case of the high-latitude ionosphere; it can only be accepted as a reasonable approximation for a very limited area. If the zenith of a receiver happens to be near the border, delineating regions with significantly different parameters of the F-region irregularities, one would expect two (and even more) peaks of $\sigma_{\chi}^{2}$. Analysis of satellite

Published by Copernicus Publications on behalf of the European Geosciences Union. 
scintillation data showed that indeed several peaks of $\sigma_{\chi}^{2}$ can occur for one satellite pass. However, the second and subsequent peaks are usually much smaller than the main one, and they were discarded in the past. This was certainly the case when Tereshchenko et al. (2005) studied joint scintillation data and SuperDARN radar measurements of plasma convection at the auroral oval latitudes. However, when the authors turned their attention to the polar cap observations at the Barentsburg station, they discovered that the latitudinal profiles of $\sigma_{\chi}^{2}$ were quite often double-peaked. Since the reason for the existence of double-peaked profiles was not certain, such events were dropped from the analysis.

This study considers data from the polar cap station Barentsburg with a special focus on cases with double-peaked latitudinal profiles of $\sigma_{\chi}^{2}$. We combine scintillation data and plasma convection measurements by the SuperDARN radars to show that such cases correspond to the onset of strongly inhomogeneous (sometimes sheared) flows near the zenith of the station and that information on additional peaks of $\sigma_{\chi}^{2}$ can be used to supplement SuperDARN data on the convection direction.

In this study we follow the Tereshchenko et al. approach by assuming that the additional peaks occur due to a change in the irregularity shape. Generally, one can expect additional peaks in the $\sigma_{\chi}^{2}$ profile if the irregularity layer is strongly inhomogeneous horizontally or very thin in height. In such cases, the undertaken approach would have limited success. Also, there is a small inconsistency in a sense that while fitting experimental data with a theoretical curve for each observed peak, the irregularity layer is assumed to be homogeneous while in reality it is not, as two (and more) types of irregularities are assumed to co-exist. The presented results below strongly suggest that despite some limitations, the Tereshchenko et al. method produces results consistent with a single-peak situation implying that the initial assumptions are acceptable.

\section{Observations}

Figure 1a, c gives examples of observations with single and double-peak latitudinal profiles of $\sigma_{\chi}^{2}$ and Fig. 1b, d shows a relationship between the orientation of the crossfield anisotropy of ionospheric irregularities and the plasma convection direction, as inferred from SuperDARN radar observations. In a single peak case of 26 January 2001, $\sim 05: 27 \mathrm{UT}$, irregularities causing scintillations were detected between $77.4^{\circ} \mathrm{N}$ and $78.2^{\circ} \mathrm{N}$ (geographic coordinates). We comment that, according to the model of satellite signal formation by Tereshchenko et al. (1999), the width of the peak in Fig. 1a is related to the degree of irregularity stretching along the magnetic field, characterized by the parameter $\alpha$, and perpendicular to it, characterized by the parameter $\beta$. The location of the peak with respect to the latitude of the receiver depends on the azimuthal orientation of the irregularities in the plane perpendicular to the magnetic field; this angle is denoted as $\Psi_{A}$. Fitting of the experimental profile for $\sigma_{\chi}^{2}$ in Fig. 1a to the model, as prescribed by Tereshchenko et al. (1999), gives $\alpha=30, \beta=5$ and $\Psi_{A}=175^{\circ}\left( \pm 5^{\circ}\right)$. This implies that, on average, the axes ratio of the irregularities is 30:5, with the major axis being aligned with the geomagnetic field. We remind the reader that the angle $\Psi_{A}$ is counted clockwise from the direction of geographic north (one cannot distinguish between the cases of $0^{\circ}$ and $180^{\circ}$ ). Comparison with a corresponding SuperDARN convection map shows reasonable agreement of the plasma flow direction and the orientation of the crossfield anisotropy of irregularities, Fig. 1b. For the convection azimuth of $\Psi_{S D}=337^{\circ}$ at the point nearest to the scintillation measurements, the difference between $\Psi_{A}$ and $\Psi_{S D}$, $\Delta \Psi=\Psi_{A}-\Psi_{S D}$, is $18^{\circ}$. Note that the considered singlepeaked event is a typical observation, especially for the auroral zone measurements over Northern Europe, where ionospheric flows are predominantly zonal and satellites travel roughly along the magnetic meridian.

For the second case of 29 September 2004, Fig. 1c, two strong peaks of $\sigma_{\chi}^{2}$ distribution are evident, at latitudes of $78.8^{\circ}$ and $78.2^{\circ}$. Fitting to the model separately for each peak gives the direction of the cross-field anisotropy of irregularities $\Psi_{A}^{(1)}=12^{\circ}\left( \pm 1^{\circ}\right)$ for the first peak and $\Psi_{A}^{(2)}=60^{\circ}\left( \pm 1^{\circ}\right)$ for the second peak. The closest SuperDARN vectors have $\Psi_{S D}=14^{\circ}\left(79.05^{\circ} \mathrm{N}, 16.38^{\circ} \mathrm{E}\right)$ and $\Psi_{S D}=69^{\circ}\left(77.99^{\circ} \mathrm{N}\right.$, $16.94^{\circ} \mathrm{E}$ ), so that the direction differences are $\Delta \Psi^{(1)}=-2^{\circ}$, and $\Delta \Psi^{(2)}=-9^{\circ}$. We note that in this case, the irregularity parameters $\alpha$ and $\beta$ are the same for the peaks, implying that within the irregularity layer there was only a change in the orientation of irregularities (plasma flow direction).

Figure $1 \mathrm{c}$ also shows an additional minor maximum in between two major ones. Its intensity is $\sim 0.13$. For this case, the orientation of the cross-field anisotropy of irregularities cannot be inferred, as the peak is much smaller than the other two.

Figure 2 presents the data for a case when three peaks in $\sigma_{\chi}^{2}$ were observed, on 7 June 2001. For this event, the irregularity shape and orientation of the cross-field anisotropy were different at the respective points of measurements, Fig. 2a, implying that the irregularity properties were changing along the satellite track. One can see that each peak of $\sigma_{\chi}^{2}$ gives the direction of the flow that corresponds quite well to the closest SuperDARN vector, Fig. 2b. The differences are $-9^{\circ}$, $+11^{\circ}$ and $-9^{\circ}$ for observations of the peak at $79.9^{\circ}, 79.6^{\circ}$, and $79.2^{\circ}$, respectively. We note that the scintillation observations for the first and second peaks were done in the area slightly away from the area of the SuperDARN measurements.

Presented data allow one to conclude that the existence of two (or more) peaks in the latitudinal distribution of $\sigma_{\chi}^{2}$ signifies that the flow direction is changing along the satellite track and the method by Tereshchenko et al. (1999, 2000a, 

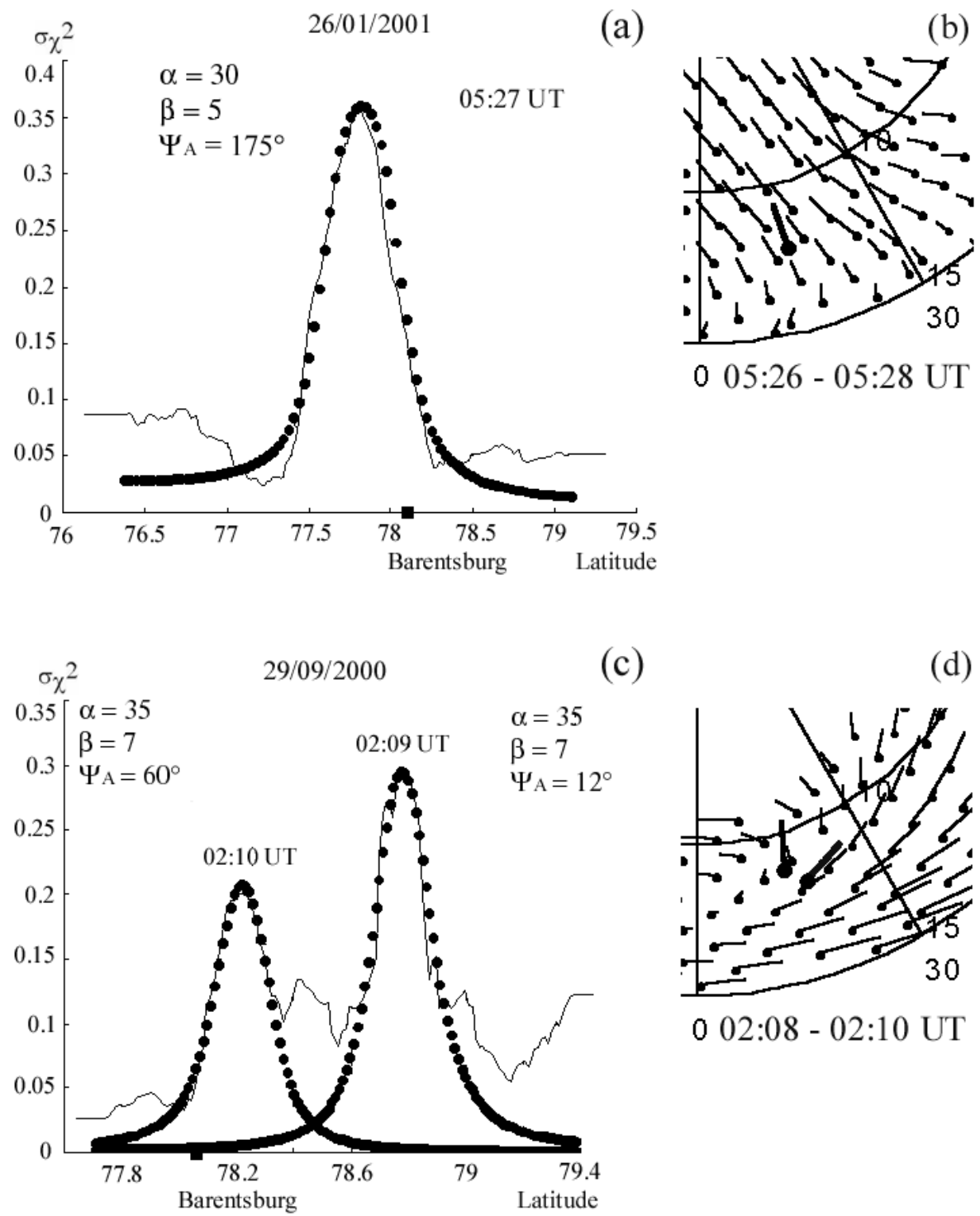

(c)

(d)

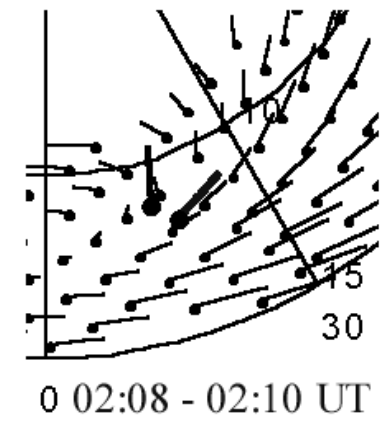

Fig. 1. (a) and (c) Two examples of observed latitudinal changes in the variance of the logarithm of the relative scintillation amplitude $\sigma_{\chi}^{2}$ (thin line) and corresponding fitted theoretical curves (dots), according to the method by Tereshchenko et al. (1999, 2000a, b, 2002, 2004, 2005). Inferred parameters of ionospheric irregularities $\alpha, \beta$ and $\Psi_{A}$ are given for every scintillation amplitude peak. Panel (a) (panel c) corresponds to a case of one (two) maximum in the distribution of $\sigma_{\chi}^{2}$. Panels (b) and (d) show SuperDARN-observed convection velocities (lines originated from dots). The geographic coordinates are used. The thick vectors on the panels (b) and (d) indicate the orientation of the cross-field anisotropy of F-region irregularities, as inferred from scintillation data.

b, 2002, 2004, 2005) is capable of detecting those changes. This means that the method can reveal large-scale details of inhomogeneous flows. In this sense, the scintillation method can be useful in supplementing SuperDARN observations.

The degree of success of the method in supplementing SuperDARN data depends on the availability of large-scale (several hundred meters) ionospheric irregularities in the areas where decameter irregularities, providing SuperDARN echoes, do not exist. Our preliminary analysis showed that the occurrence of strong scintillations correlates with the occurrence of SuperDARN HF echoes, in agreement with Mi- lan et al. (2005). However, quite often only either large-scale irregularities or decameter irregularities exist, so that either only scintillation or SuperDARN data are available.

We showed the event with partial radar and satellite data overlap in Fig. 2. In Fig. 3a we present an example when two peaks in $\sigma_{\chi}^{2}$ were detected, on 23 June 2002. Both peaks were observed in the area with no SuperDARN data at the time of scintillation measurements, Fig. 3b. The next SuperDARN 2-min scan does have data in the area of the satellite measurements made earlier, and the convection vectors agree well with the satellite-inferred direction of the convection, 
1728 E. D. Tereshchenko et al.: VHF scintillations, orientation of anisotropy, direction of plasma convection in the polar cap
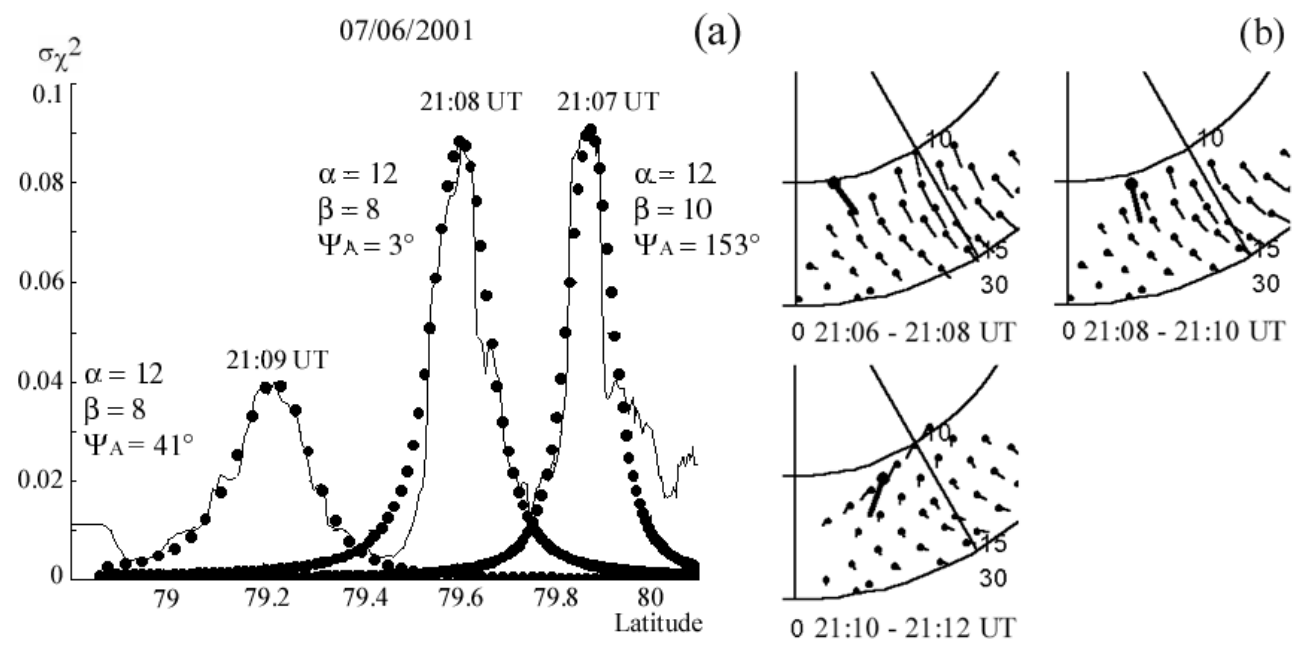

Fig. 2. The same as in Fig. 1 but for the event of 7 June 2001.
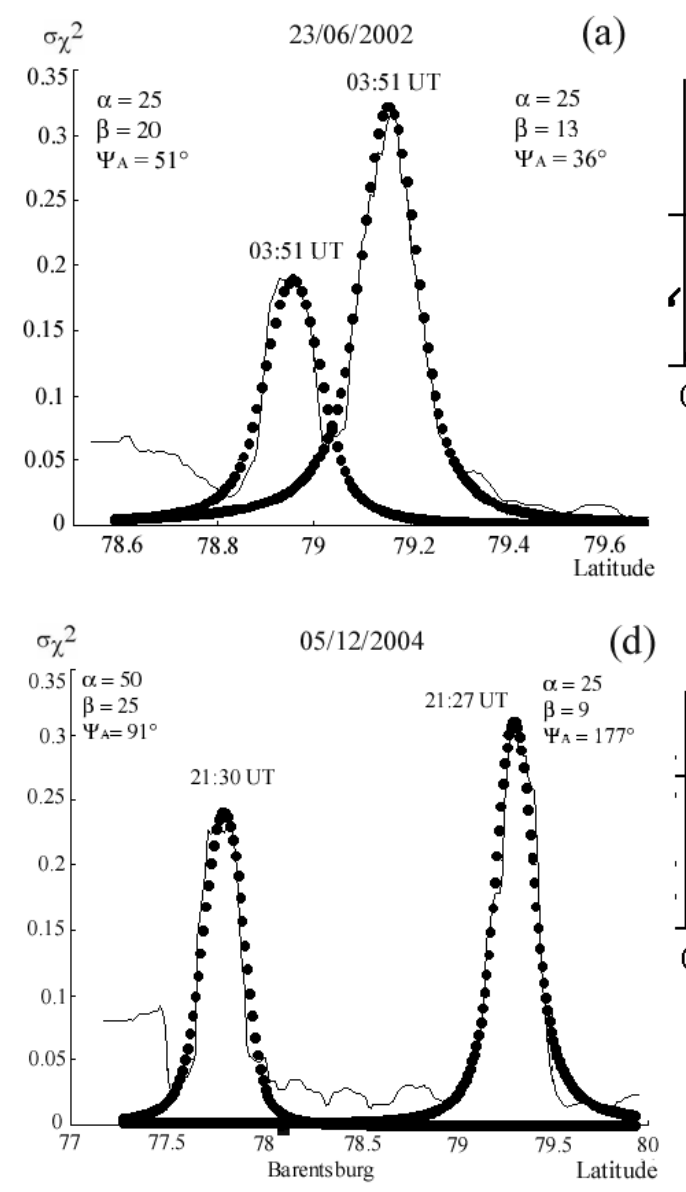

(b)

(c)

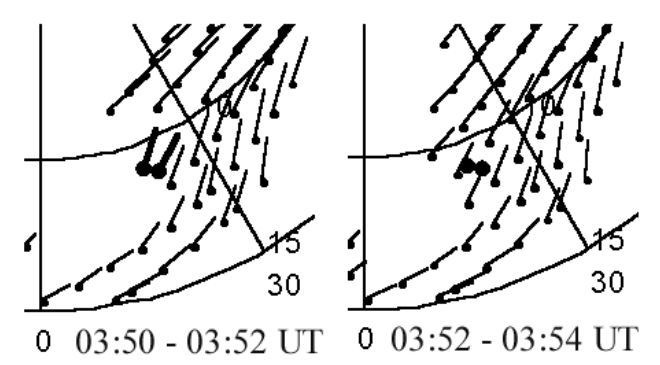

(d)

(e)

(f)

\section{(b)}

. 

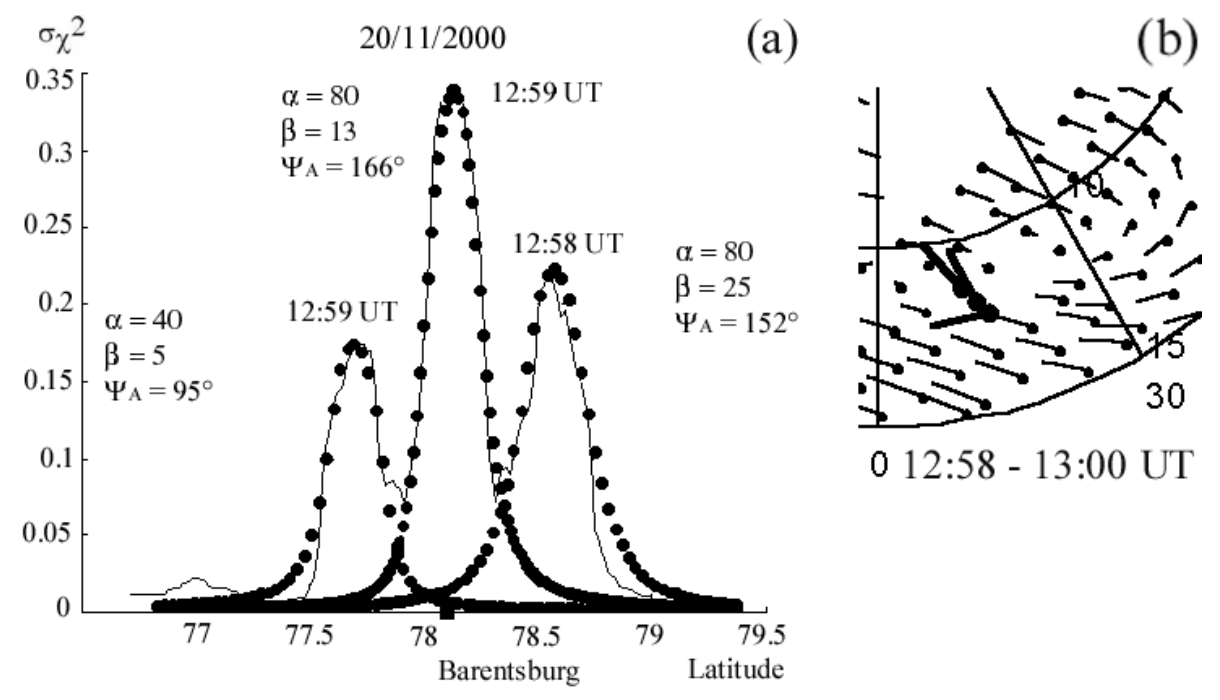

Fig. 4. The same as in Fig. 1 but for the case of 20 November 2000.

each peak separately to the model gives $\Psi_{A}=177^{\circ}\left( \pm 1^{\circ}\right)$ for the first peak and $\Psi_{A}=91^{\circ}\left( \pm 1^{\circ}\right)$ for the second one. $\Psi_{A}$ values differ significantly, suggesting that the plasma convection direction was quite different at the considered locations. Figure $3 \mathrm{~d}$, f compares the satellite-inferred convection direction and SuperDARN convection map. SuperDARN measured $\Psi_{S D}=3^{\circ}$ and $\Psi_{S D}=288^{\circ}$, so that the direction differences are $\Delta \Psi^{(1)}=6^{\circ}$ and $\Delta \Psi^{(2)}=-17^{\circ}$, respectively, in good agreement with the orientation of the cross-field anisotropy of irregularities. Notice that according to the SuperDARN convection map, the satellite was crossing the local reversal in the plasma flow and the two directions of the flow were "captured" by the method quite well. However, one would expect an additional, intermediate maximum between the two major ones, as the SuperDARN map clearly shows a rotation of the flow and one would expect another peak in $\sigma_{\chi}^{2}$ in between the two major peaks. No strong scintillations were detected in this area and no convection direction estimates from the scintillation data are available because $100-300 \mathrm{~m}$ irregularities were not excited in this area. We note here that one would not expect a one-to-one occurrence of VHF scintillations and HF echoes because, generally, while large-scale $\sim 300 \mathrm{~m}$ irregularities responsible for VHF signals might be related to structured particle precipitation into the ionosphere and further nonlinear evolution of plasma "clouds", the decametre irregularities monitored by the SuperDARN radars are very likely formed through plasma instabilities generated at "the boundaries" of plasma clouds (Tsunoda, 1988).

The last considered event hints clearly at the reason for the more frequent occurrence of double/multiple peaks in the polar cap scintillation data; the flow reversal and the centers of large-scale (and more local) vortices often occur mostly at these high latitudes. For observations in the auroral zone, the flow is predominantly zonal and double (multiple) peaks are seen seldom.
One might generally think that since satellite data are not as frequent and limited in space as SuperDARN data, the advantage of involving the scintillation data for overall convection maps is not great, and this is certainly true. We would like to point out that there are special occasions when the scintillation data could be of greater value. In Fig. 4 we demonstrate observations for the event of 20 November 2000. One can see that the satellite data with three peaks are available exactly in the "eye" of a large-scale vortex seen by the radars at its peripheral part but not in the eye area.

Once confidence was achieved that the observed double/multi-peaked profiles of $\sigma_{\chi}^{2}$ simply reflect the existence of strongly inhomogeneous or sheared flows in the polar cap ionosphere, statistics for the differences between the cross-field orientation of irregularities, as inferred from scintillation data and plasma convection direction, as measured by SuperDARN $(\Delta \Psi)$, were compiled, Fig. 5. We included cases of single- and double-peak observations. The total number of points is 104 , which is a significantly larger statistics than what was available in the previous comparison of Tereshchenko et al. (2005). Data are binned in $20^{\circ}$ steps. One can see that the vast majority of points are located within an interval of $-20^{\circ}$ to $+20^{\circ}$, and the number of larger deviations decreases with $\Delta \Psi$ increase.

\section{Summary and conclusion}

In this study we showed that the variance of the logarithm of the relative amplitude of VHF scintillations recorded at the polar cap station Barentsburg often has two and more peaks in its latitudinal profile. Comparison with SuperDARN convection data showed that these peaks reflect a strong rotation of the flow in the area of scintillation observations. The differences between the orientation of the F-region anisotropic 
1730 E. D. Tereshchenko et al.: VHF scintillations, orientation of anisotropy, direction of plasma convection in the polar cap

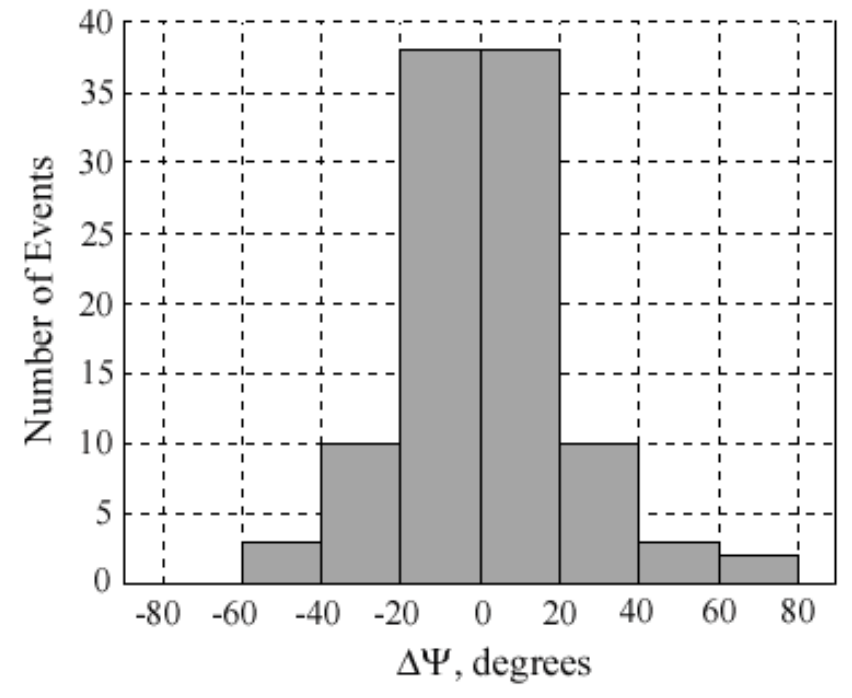

Fig. 5. Histogram distribution for the difference between the direction of the plasma flow, according to SuperDARN measurements, and the orientation of cross-field anisotropy, according to scintillation measurements at Barentsburg (Spitsbergen Archipelago). Data include scintillation receiver detection of single and double-peaks in the latitudinal profile of $\sigma_{\chi}^{2}$. Total number of events is 104 .

irregularities in the plane perpendicular to the magnetic field and the $\boldsymbol{E} \times \boldsymbol{B}$ flow direction, as inferred from SuperDARN data, were found to be small, mostly within $\pm 20^{\circ}$, for the 104 events investigated. The comparison provides evidence that the scintillation data can be used to monitor the direction of not only large-sale flows but also some of its details, and the scintillation observations can successfully supplement SuperDARN data on the direction of plasma flow at high latitudes.

Acknowledgements. The authors are grateful to G. E. Kadotchnikov for the help in SuperDARN data processing and to B. Z. Khudukon, Yu. A. Mel'nichenko, A. Roskuljak and S. Shadrin for their help in satellite signal measurements. This work was supported by the NSERC (Canada) grant to A. V. Koustov, by the Russian Foundation of Fundamental Research grant no. 06-05-64540 and government contract no. 02.518.11.7061 with the Federal Agency for Science and Innovations of the Ministry of Education and Science of the Russian Federation.

Topical Editor M. Pinnock thanks two anonymous referees for their help in evaluating this paper.

\section{References}

Aarons, J.: Global morphology of ionospheric scintillations, Proc. IEEE, 70, 360-378, 1982.

Eglitis, P., Robinson, T. R., Rietveld, M. T., Wright, D. M., and Bond, G. E.: The phase speed of artifical field-aligned irregularities observed by CUTLASS during HF modification of the auroral ionosphere, J. Geophys. Res., 103, 2253-2259, 1998.
Fremouw, E. J., Rino, C. L., Livingston, R. C., and Cousins, M. C.: A persistent subauroral scintillation enhancement observed in Alaska, Geophys. Res. Lett., 4, 539-542, 1977.

Gailit, T. A., Gusev, V. D., Ivanov, M. I., and Perekalina, E. O.: Differential-phase study of the anisotropy of ionospheric irregularities, Geomag. Aeron., 22, 622-625, 1982.

Martin, E. and Aarons, J.: F layer scintillations and the aurora, J. Geophys. Res., 82, 2717-2722, 1977.

Milan, S. E., Basu, S., Yeoman, T. K., and Sheehan, R. E.: A comparison of satellite scintillation measurements with $\mathrm{HF}$ radar backscatter characteristics, Ann. Geophys., 23, 3451-3455, 2005 , http://www.ann-geophys.net/23/3451/2005/.

Moorcroft, D. R. and Arima, K. S.: The shape of the F-region irregularities which produce satellite scintillations - evidence for axial asymmetry, J. Atmos. Terr. Phys., 34, 437-450, 1972.

Rino, C. L., Livingston, R. C., and Matthews, S. J.: Evidence for sheet-like auroral ionospheric irregularities, Geophys. Res. Lett., 5, 1039-1042, 1978.

Rino, C. L. and Livingston, R. C.: On the analysis and interpretation of spaced-receiver measurements of transionospheric radio waves, Radio Sci., 17, 845-854, 1982.

Tereshchenko, E. D., Khudukon, B. Z., Kozlova, M. O., and Nygren, T.: Anisotropy of ionospheric irregularities determined from the amplitude of satellite signals at a single receiver, Ann. Geophys., 17, 508-518, 1999, http://www.ann-geophys.net/17/508/1999/.

Tereshchenko, E. D., Khudukon, B. Z., Kozlova, M. O., Evstafiev, O. V., Nygren, T., Rietveld, M. T., and Brekke, A.: Comparison of the orientation of small scale electron density irregularities and $\mathrm{F}$ region plasma flow direction, Ann. Geophys., 18, 918926, 2000a, http://www.ann-geophys.net/18/918/2000/.

Tereshchenko, E. D., Kozlova, M. O., Evstafiev, O. V., Khudukon, B. Z., Nygren, T., Rietveld, M., and Brekke, A.: Irregular structures of the F layer at high latitudes during ionospheric heating, Ann. Geophys., 18, 1197-1209, 2000b, http://www.ann-geophys.net/18/1197/2000/.

Tereshchenko, E. D., Romanova, N. Yu., and Kozlova, M. O.: Comparison of the cross-field anisotropy of small-scale irregularities with ionospheric convection model, Geomag. Aeron., 42, 432436, 2002.

Tereshchenko, E. D., Kozlova, M. O., Kunitsyn, V. E., and Andreeva, E. S.: Statistical tomography of subkilometer irregularities in the high-latitude ionosphere, Radio Sci., 39, RS1S35, doi:10.1029/2002RS002829, 2004.

Tereshchenko, E. D., Romanova, N. Yu., and Koustov, A. V.: Orientation of the cross-field anisotropy of small-scale ionospheric irregularities and direction of plasma convection, Ann. Geophys., 23, 1227-1237, 2005, http://www.ann-geophys.net/23/1227/2005/.

Tsunoda, R. T.: High latitude irregularities: A review and synthesis, Rev. Geophys., 26, 719-760, 1988. 